\title{
PERFECT LINE SIMPLIFICATION FOR VISUALIZATION IN DIGITAL CARTOGRAPHY
}

\author{
Sham Prasher \\ School of Information Technology and Electrical Engineering \\ University of Queensland \\ sham@itee.uq.edu.au
}

\begin{abstract}
Spatial data is often voluminous and, as a result, difficult to process or manipulate efficiently. In digital cartography spatial data is presented to provide accurate information regarding real-world geography. However because available data may often exceed that which is needed, techniques for the reduction of the data load have long been a focus of study. Cartographic generalization looks at reducing the amount of map data while preserving the quality of visually conveyed information. We look at current techniques of cartographic generalization, simplification algorithms in particular, and show their dependence on unreliable geometric properties can cause imperfect results. We introduce an algorithm that produces visually perfect simplifications of spatial objects in linear time where previous algorithms have failed to do so. We show how the algorithm can be used with standard co-ordinate data and as well as z-value data.
\end{abstract}

Keywords: simplification, cartographic generalization, spatial data

\section{INTRODUCTION}

Generalization is the process of reproducing images in reduced versions whilst maintaining as much visual quality and convey as much meaning as the original. Similarly cartographic generalization is the process of reproducing maps at smaller scales (i.e. different levels of detail) while retaining as much information as the resulting image will allow. Where cartographers traditionally performed this task manually, the advent of 
modern computing power and geographic information systems has brought promise to automating the process.

Automatic cartographic generalization techniques have been the focus of study for the past 30 years especially with the recent growth of geographic information systems. The aim of cartographic generalization is to derive small scale, less detailed, maps from larger scale maps. Automated generalization of spatial information should simulate the work of the human cartographer [9]. This condition poses the greatest challenge in all attempts of automation. The reason is that processes that allow human vision to distinguish relationships between buildings, rivers, roads and other topographic elements are difficult to identify and classify, let alone computerize. With spatial data becoming more widespread there is a growing need for efficient data manipulation (generalization) to satisfy various commercial uses. This need is particularly high in online systems where the processing and retrieval time of data are correlated with quality of service.

Typically spatial data are stored as images in either raster or vector format. The raster format stores the content of each pixel of an image individually. Storage is higher but the image is complete and ready for display. Spatial databases are commonly used to store and manage vector data. Vector data includes only information on objects within the image that are put together to form a complete picture. Storage requirements are lower but some computation is needed to assemble the picture. Vector data (points) are usually stored in some co-ordinate system format such as $(\mathrm{x}, \mathrm{y})$ grid values, longitude-latitude or Lambert Azimuthal coding for geographic data. Alternatively z-values [12], which are generated from the regular decomposition of space, can also be used to describe spatial data. The development of efficient ways to encode, index and manipulate $z$-values has proven them useful in many spatial database applications. Although we look at the possible use of $z$-values, a full explanation of them goes beyond the scope of this paper.

In our work the vector format is preferred because it allows for easier image manipulation and can be cached more readily for future use, such as zooming and panning by using cached data.

Generalising maps has thus far proven non-trivial. Like many other tasks it requires complex human reasoning regarding the visual and topological relationships between multiple elements. Cartographic generalization deals with making sense of spatial data at the macro level. As a result numerous processes are employed to make an image that may be overloaded with detail, visually legible during generalization. Examples include amalgamation and smoothing. Non-spatial operators like SQL 'selection' statements on spatial layers can also be used to remove unwanted clutter 
such as 'all minor roads'. An important factor in automated generalization is deciding which techniques, if any, to use or are necessary. The choice usually depends on context and exactly what kind of result is desired. Different kinds of manipulation can mean different information is presented in the final image. The scope of this paper takes the approach of geometric generalization [7]. The focus is solely on simplification of elements within a map at the micro level. The issue here is reducing the amount of data required to represent objects in an already legible image.

Our goal is to produce simplifications of cartographic objects, stored as spatial data, that look as close as possible to their originals. For this purpose we introduce the concepts of perfect generalization and minimal perfect generalization (PG/MPG). In the section 2 we provide an overview of research to date in this area. Section 3 introduces the concepts of PG and MPG. Section 4 outlines a methodology for PG. The paper is concluded, and notes on future work are given in section 5 .

\section{BACKGROUND}

Generalized images are usually produced with a certain level of accuracy. With current techniques not being perfect, this level may vary between situations and is what warrants the utilisation of several different correctional and supporting techniques. Among several different approaches to the problem, line simplification algorithms are one of the most fundamental. A number of such algorithms have been developed and refined over the past few decades. One of the most widely used, the Douglas Peucker algorithm [3] works by initially building a baseline connecting the first and last points. The distance of a constructed line joining each point to the baseline perpendicularly is measured as the selection factor. The point with the longest distance is kept and included in a new baseline. The process iterates until a set number of points are selected or points fail to exceed some pre-defined threshold distance from the baseline.

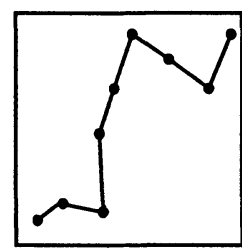

(a) Original line.

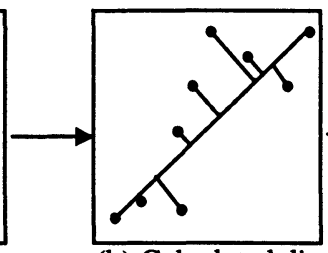

(b) Calculated distances from baseline.

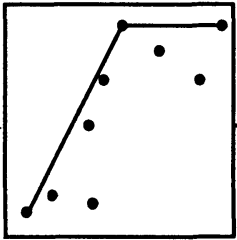

(c) Baseline after 1 point is added.

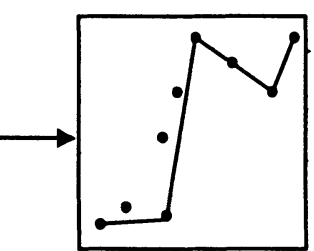

(d) Baseline after 3 points are added.

Figure 1 Douglas Peucker Line Simplification 
A similar approach is taken by the line-length [1] and angular-change [9] algorithms. The first eliminated points iteratively based on how little difference their absence makes. This difference is measured as the distance between a point's two (previous and next) neighbouring points. The point whose neighbours form the shortest line is eliminated. Similarly the angularchange algorithm eliminates points based on what change of angle neighbouring points will produce. The notable issue with these is that their criteria alone cannot determine the actual shape of the line or guarantee the accuracy of the result. Taking it one step further, the Visvalingam Whyatt [13] algorithm calculates area changes before and after point elimination. Again this single criterion is not very useful, as distance and line shape cannot be derived from a single area measure. Like the line-length and angular-change approaches this algorithm makes an assumption that the minimal measure of its metric (i.e. area displacement) correlates to a minimal change in the polyline's shape.

Individually these algorithms are easily tested based on how well their results resemble the original image. However testing between algorithms, and determining which is the best, is difficult because each is usually evaluated using different metrics [14]. As with the Douglas Peucker algorithm these techniques do not always preserve the original shape of objects. The main reason the shape is often compromised is the algorithms only consider the vector properties of line elements meaning that no consideration is given to how the simplification will affect the look of the data once mapped to a digital display. This also contributes to the reason why conversions between vector and raster formats are error-prone. Although there are a number of refining and smoothing techniques [10] that are designed to handle such after-effects, they are themselves imperfect and still cannot completely prevent the chance for error.

Another algorithm designed by $\mathrm{Li}$ and Openshaw [8] considers the equivalent raster representation of given vector data during the simplification process. The algorithm uses the concept of the Smallest Visible Object (SVO) as the finest cartographic detail visible by a human user. In raster format this equates to a single pixel. A list of points is first given as the polyline to be simplified. Then a layer of pixels is placed over the vector data. The pixels are defined at the resolution of the digital environment onto which the resulting simplified data is to be displayed. Wherever a pixel contains a point from the given list, the centre of that pixel is returned as a new point in the simplified line, which is then used to represent all points within that pixel. Another difference is that new points are returned, not one from the original list. 


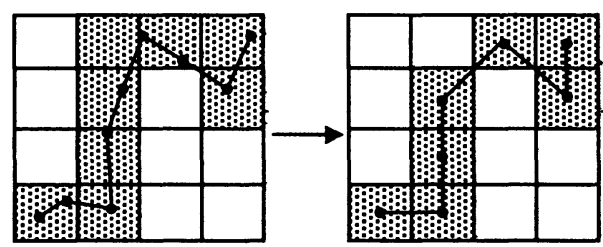

(a)

(b)

Figure 2 (a) The original image rendered to pixels (represented as shaded blocks) and (b) the Li Openshaw simplification of the same data rendered at the same resolution.

There is no guarantee the original shape is preserved because newly created points govern the simplified line's shape (3b), not original ones. Revisiting the Douglas Peucker algorithm we can see how inaccuracies can occur. Given vector displacement as a point discriminator there is no particular vector distance (from the baseline) that is suitable for all points at that resolution. If this distance, or tolerance, was set to lower than a resolution's pixel width then the simplified line could be identical to the original. It also does not consider cases where points within this tolerance are located in different pixels because the baseline itself passes close to a pixel border. The other geometry-based algorithms suffer the same drawback.

Other trends in digital cartography include scale-indexing techniques [5] that take a paradigmatically opposite approach by using pre-processing to construct indexes. This allows easy scale-based access to data instead of employing on-the-fly generalization algorithms. The issue with using such methods is that pre-computation cannot easily anticipate, therefore accommodate all possible uses of the data by users, which themselves may change. To accommodate large changes often recomputation of the entire index is required.

An important goal of generalization is to preserve topology that often can convey most of an image's meaning. If these relationships cannot be shown given a purely geometric transformation using polyline simplification, other techniques mentioned earlier can be used. Smoothing, amalgamation, exaggeration, collapse and displacement are common ways of modifying the resulting image to convey important or relevant information. Works presented in [6][15] discuss criteria that must be satisfied in order to guarantee some level of quality from the generalization. The involvement of these techniques also usually calls for manual intervention and so has led to the conceptualisation of large complex frameworks [10, 2, 11] for generalising. This can complicate things more than is desirable, involving additional processes, time and resources to what may be intended as an efficient procedure. One evaluation method [6] in particular uses a similar 
concept to the Li Openshaw algorithm for correctness checking. It works by overlaying a gird on the result and measuring whether the generalization process was done consistently throughout the original map by comparing levels of 'clutter' within each grid cell. In this paper we focus on simplification and avoid additional 'correctional' techniques that may add unnecessary complexity. Our work builds on the concept of overlaying a raster grid of pixels over vector spatial data to serve as a guide for simplification.

\section{DEFINITION OF PG AND MPG}

The aim of PG/MPG is to generate a set of spatial data, which is visually indistinguishable, in a digital environment, to the larger original dataset on which it was based. Therefore it can provide exactly the same visual experience at a fraction of the storage requirement and, hence, transmission time or processing cost.

First we must clarify the use of scale and resolution. Scale is the term used by cartographers to state how far reduced elements on a map are from their real life counterparts. Since maps are reduced images of real life areas the scale factor can reach well into the millions. A small scale corresponds to a highly detailed map whereas a large scale denotes lower detail. The term resolution is often used interchangeably with scale, sometimes to the point of confusion. In this paper resolution is used to the detail at which some display hardware (i.e. CRT/LCD) renders spatial data. A high or low resolution corresponds to high or low display detail respectively.

When data, stored at a particular scale, is rendered to some physical display there is no guarantee that the scale and screen resolution are exactly match. That is if the screen resolution is low then only some of the data is made physically visible (figure 4). Conversely at high resolutions a larger amount of data can be handled and viewed. Ideally some automated decision-making would be used to determine the best amount of data to use and display at a particular resolution. This leads to the concept of perfect generalization.

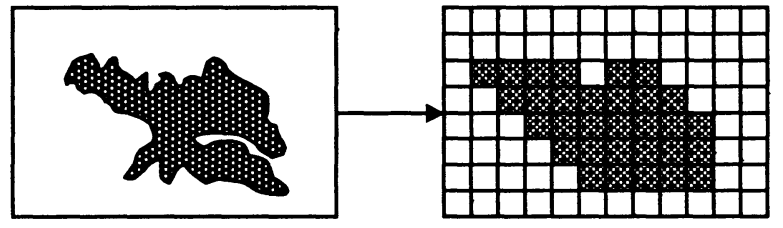

Figure 3 Original data mapped to a low resolution.

If a simplification algorithm modifies a dataset and that modified data set renders to the computer screen an identical picture to that which is rendered 
by the original dataset, then that simplification algorithm is said to be perfect. More precisely, given a set of data $d$ that is rendered to a screen at a particular resolution $r$ an image $i$ is produced. We note this as $\operatorname{ren}(d, r)=i$. If $d$ is initially generalised by some simplification method to a reduced data set $d^{\prime}$ then rendered to $r$ another image, $i$ ' is produced; $r e n\left(d^{\prime}, r\right)=i$ '. If $i$ l looks exactly the same as $i,\left(i{ }^{\prime}=i\right)$, using the same display and resolution, then the generalization is said to be perfect. More formally, if $d^{\prime} \subseteq d \wedge i=i^{\prime}$. In some situations it may be necessary to replicate the same visual effect of an image, as much as possible, at a lower resolution. The change equates to a transition from a high to a low-resolution image requested by a user or forced by some hardware restrictions such as a low resolution WAP screen. Perfect simplification occurs when every pixel rendered in the original image is represented by some 'equivalent' lower resolution pixel on the output device. Also no pixels at the lower resolution are rendered that are not represented by some pixel in the original image. This follows the definition $d^{\prime} \subseteq d \wedge i=i^{\prime}$ as closely as the output resolution is able. Section 5.2 covers this point in more detail.

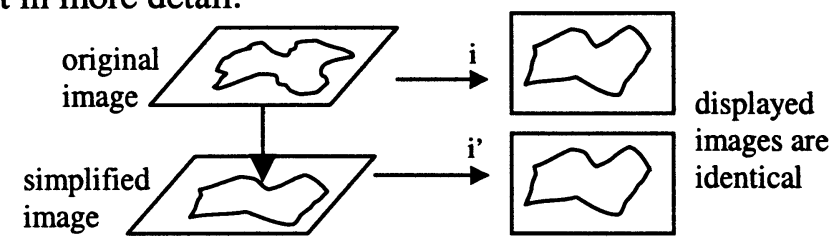

Figure 4 Perfect generalization.

The problem with perfect generalization is that an algorithm that takes a vector object and returns the same vector object is considered ideal or perfect. The concept of minimal perfect generalization is introduced to express the fact that an algorithm is preferable if it greatly reduces the size of data needed to represent an object. Minimal perfect generalization refers to a generalization that uses the minimal number of points of $d$ to construct a perfect generalization $d^{\prime}$.

So if $\left(d^{\prime} \subseteq d \wedge i=i^{\prime}\right) \wedge\left(\neg \exists p, p \in d^{\prime} \bullet\left(d^{\prime}-p\right)=d^{\prime \prime} \wedge \operatorname{ren}\left(d^{\prime \prime}, r\right)=i{ }^{\prime \prime}=i\right)$ then $d^{\prime}$ is minimal.

\section{PERFECT GENERALIZATION ALGORITHM}

\subsection{Assumptions and notations}

For data storage we use a single relational table containing one field for object IDs and another for the object's geometry. We specify the geometry of a single object as a sequence of spatial $(x, y)$ coordinates using Oracle's 
SDO_GEOMETRY spatial data type. Alternatively points could be stored as z-values using quadtree coding [12]. The object may be closed (polygon) or open (polyline). An index is placed on the table to allow spatial querying'.

We must make note that the data structures and algorithms provided in this paper serve only to provide an example of the PG and MPG methods as applied to data points. Since such structures are usually defined based on what queries are expected of the database [4] they are subject to change with context.

Given a spatial object $O$ that is comprised of a set of points in 2D space; i.e. $O=\left\{\mathrm{t}_{1} \ldots \mathrm{t}_{\mathrm{k}}\right\} . O$ may represent either a closed polygon or an open polyline. In either case points can be retrieved in a linear sequence. A function $F$ applied to $O$ will generate an object $O^{\prime}$ comprised of a set of points such that $F(O)=O^{\prime}=\left\{\mathrm{q}_{1} \ldots \mathrm{q}_{\mathrm{m}}\right\}$ where $O^{\prime} \subseteq O$. In other words $F(O)$ is a simplification of $O$.

The purpose of the MPG is to render the original object to a display at a given resolution using only a fraction of its data. Therefore we must consider the display of objects in terms of resolution. A small resolution is limited to low detail whereas high resolution allows for high detail. A resolution $r$ is required for the original object $O$ to be rendered with no visual loss of detail. To render $O$ to some display with a resolution $r^{\prime}$, such that $r^{\prime}<r$, with no visual loss of detail, $O$ can be generalised to object $O^{\prime}$ to reduce the amount of needed data. First $r$ must be mapped to $r$ ':

Using $r$ we can define a resolution $r=\left[r_{x} \times r_{y}\right]$; eg 640 $\times 480$. The range of $r$ must cover the spatial extent of some digital display (such as an LCD screen), which can be given as $\left[e x t_{x} \times e x t_{y}\right]$ and is specified in some metric measurement. The points in $O$ cover a spatial range $s r=\left[s r-x_{\max }, s r-y_{\max }, \mathrm{sr}-\right.$ $\left.\mathrm{x}_{\min }, \mathrm{sr}-\mathrm{y}_{\min }\right]$ such that $0 \leq\left[\mathrm{sr}-\mathrm{x}_{\max }, \mathrm{sr}-\mathrm{x}_{\min }\right] \leq$ ext $x_{x}$ and $0 \leq\left[\mathrm{sr}-\mathrm{y}_{\max }, \mathrm{sr}-\mathrm{y}_{\min }\right] \leq$ ext $y_{y}$. Each point in $O$ has spatial coordinates $(x, y)$ such that $0 \leq x \leq e x t_{x}$ and 0 $\leq y \leq e x t_{y}$.

The resolution $r$ is made up of a grid of pixels. Each pixel $p$ in $r$ has its own spatial extent. That is $\forall p \in r \Rightarrow p_{\text {ext }}=\left[\mathrm{x}_{\max }, \mathrm{y}_{\max }, \mathrm{x}_{\min }, \mathrm{y}_{\min }\right]$ such that $0 \leq$ $\left[\mathrm{x}_{\max }, \mathrm{x}_{\min }\right] \leq e x t_{x}$ and $0 \leq\left[\mathrm{y}_{\max }, \mathrm{y}_{\min }\right] \leq e x t_{y}$. Each pixel essentially covers a square area having four edges and four vertices ${ }^{2}$. The latter is drawn from the pixel's spatial extent and is given as $\left[p-x_{\max }, p-y_{\max }\right]$, [p- $\left.x_{\min }, p-y_{\min }\right]$, [p$\left.\mathrm{x}_{\min }, \mathrm{p}-\mathrm{y}_{\max }\right],\left[\mathrm{p}-\mathrm{x}_{\max }, \mathrm{p}-\mathrm{y}_{\min }\right]$. Vertices and edges may belong to multiple neighbouring pixels.

The width length of a pixel $s$ is given by $\left(e x t_{x} / r_{x}\right)$. The ext $t_{y}$ does not need to be included because it is assumed that pixels cover square areas and that a given resolution is always properly scaled to fit the display area. Therefore

\footnotetext{
${ }^{1}$ The exact index technique is not vital to the paper's focus; for instance we assume an RTree is used.

${ }^{2}$ While a pixel is a dot we represent its extent as the square area covered by its dimensions.
} 
$\left(r_{x} \times s\right)=e x t_{x}$ and $\left(r_{y} \times s\right)=e x t_{y}$. For example if ext $=1000$ and $e x t_{y}=500$, and $r_{x}=500$ then $r_{y}$ must be 250 .

Next $r$ must be converted to the desired resolution $r^{\prime}$. To do this we define $\left[r_{x}^{\prime}, r_{y}^{\prime}\right]$ on $r^{\prime}$ such that $r_{x}^{\prime}<r_{x}$ and $r_{y}^{\prime}<r_{y}$ and $\left(r_{x}^{\prime} \times s\right)=e x t_{x}$ and $\left(r_{y}^{\prime}\right.$ $\times s)=e x t_{y}$. The desired resolution spans the extent of the display because the number of pixels is reduced hence they are a different size. In $r$ ' a pixel's width is given by $\left(e x t_{x} / r_{x}^{\prime}\right)$.

(a)

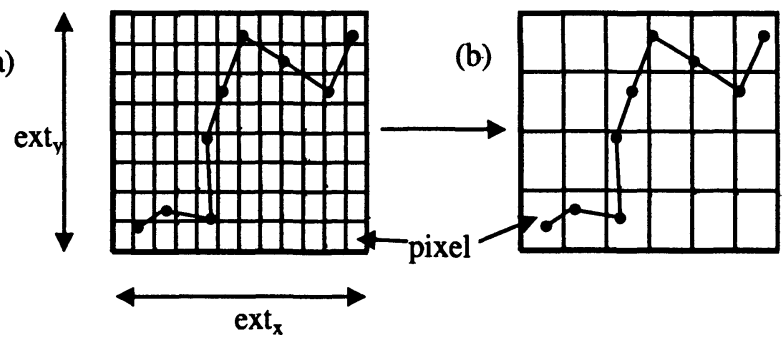

Figure 5 Grid over object $\mathrm{O}$ at resolution $\mathrm{r}(\mathrm{a})$ and $r^{\prime}(\mathrm{b}){ }^{3}$

Pixels in $r^{\prime}$ have the same properties as those in $r$ except they cover different spatial extents.

\subsection{Method}

The preliminary step gives us a foundation similar to that used by the $\mathrm{Li}$ Openshaw algorithm. Knowing how the spatial data would appear on display serves as the guide to simplifying $O$ into $O^{\prime}$. In the following algorithm we use a transform function on spatial $(\mathrm{x}, \mathrm{y})$ co-ordinates to transform the resolution of data points to that of the overlaid grid. We define another function truncate that performs the same transformation except on z-values. The function can be used for both simplification at the same resolution or simplification to a lower resolution as mentioned in section 3. Like the $\mathrm{Li}$ Openshaw method, the PG algorithm requires a single pass through the point sequence thus operating in $O(n)$ time.

\section{Algorithm Parameters:}

$Q:$ A single object of the data table in the form ObjectID, geometry $(x 1, y 1, \ldots$ , xn,yn).

$\operatorname{ObjectID}(x)$ : The $x^{\text {th }}$ point in the object's geometry.

$R$ : An empty object of the form $\operatorname{ObjectID,~geometry~}(x 1, y 1, \ldots, x n, y n)$.

\footnotetext{
${ }^{3}$ We show the grid of resolution $r$ at a slightly coarser level than it actually is for legibility. Realistically $r$ would be set at a finer level in order to describe the polyline in the illustration.
} 
transform $(a)$ : Function that changes a point $a$, specified at the original resolution $r$, to its equivalent value $b$, which is specified at a lower resolution $r^{\prime}$. For example given $r=[1000 \times 500], r^{\prime}=[500 \times 250]$ and $a=(500,250)$ then $b=(250,125)$.

truncate $(a, x)$ : Function that removes the $x$ rightmost digits of a. z-value $a$ thus changing $a$ to a z-value of a larger scale. This function can be used interchangeably with transform $(a)$ if $\mathrm{z}$-values are used instead of $(\mathrm{x}, \mathrm{y})$ coordinates.

\section{Algorithm: Perfect Generalization}

\section{begin}

//put the first point of $Q$ into $R$

$R=$ ObjectID(1);

For each subsequent point $\mathrm{i}$ :

//check if the point is in the same pixel as the previous point if transform $(i)=$ transform $(i-1)$ then

//if the next point is not in the same pixel keep this point if transform $(i+1) \neq$ transform $(i-1)$

$$
R=R+(\operatorname{ObjectID}(i))
$$

else

$$
R=R+(\operatorname{ObjectID}(i))
$$

end if

loop

go to next point $i=i+1$

Check if first point can be removed, joining its predecessor and successor. end

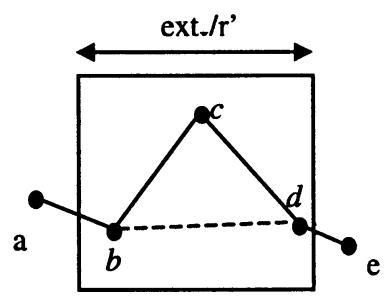

Figure 6 From the algorithm point $c$ is not needed and is removed, linking $b$ to $d$.

Using z-values allows quick conversion from one resolution to another. The drawback of $z$-values is they can only be truncated to a discrete number of specific resolutions. Each digit truncated sets the value to a different resolution. If the display resolution $r$ does not match one of these resolutions then the result may have to be stretched or condensed slightly to fit the output display.

To begin with the first point is kept as a starting point. The algorithm eliminates points that form lines within a pixel since their inter-connections 
become insignificant when the whole pixel is rendered. Lines that enter or exit different pixels, i.e. the endpoints occur in two different pixels after transformation, are kept. The reason is that these lines' directions contribute to preserving the image's overall appearance by determining which pixels are covered or crossed by the object, as follows:

1. The direction of lines entering and exiting two different pixels is determined by the position of their endpoints within those pixels.

2. Given 3 points $A, B$ and $C$ the lines $A B$ and $B C$ are formed. The elimination of the exiting point $B$, forming a new line $A C$, produces no change if $A C$ does not cross different pixels than those crossed by $A B$ and $B C$. Otherwise the visualization of $A C$ is different to $A B C$.

3. We denote the set of pixels crossed by a line $l$ as $\mathrm{P}(l)$. Therefore elimination of point $\mathrm{B}$ is where $\mathrm{P}(A C)=\mathrm{P}(A B) \oplus \mathrm{P}(B C)$.

4. A line $l$ crosses a pixel edge or vertex at a specific point called an intersection point or IP. ${ }^{4}$

5. An intersection point, being on a border can touch multiple pixels. For a given IP these pixels are denoted $\mathrm{P}(\mathrm{IP})$.

6. Given any pixel and some point IPx occurring on its border there can be up to eight different instances of $\mathrm{P}(\mathrm{IP} x)$, four for the sides and four for each vertex of the pixel. If we move an IP $x$ along a pixel border we can see that encountering any vertex will cause a change in the set $\mathrm{P}(x)$.

7. For any 2 intersection points $I P 1$ and IP2 where $P(I P 1)=P(I P 2)$ they must lie on either the same pixel side or vertex.

8. Conversely for any IP1, IP2 where $\mathrm{P}(\mathrm{IP} 1) \neq \mathrm{P}(\mathrm{IP} 2)$ then one of the following must hold:

- IP1 is on a pixel edge $e 1, \mathrm{IP} 2$ is on a pixel edge $e 2$ and $e 1 \neq e 2$.

- IP1 is on any pixel edge, IP2 is on any pixel vertex.

- IP2 is on any pixel edge, IP1 is on any pixel vertex.

9. Using the lines $A B, B C, A C$ the instance of a pixel vertex occurring on the edge of or within the triangle $A B C$ denotes that $\mathrm{P}(A C) \neq \mathrm{P}(A B) \oplus$ $\mathrm{P}(B C)$.

10. If a pixel vertex is not found in the triangle $A B C$ then $A C$ touches on or passes through the same pixels as the polyline $A B C$.

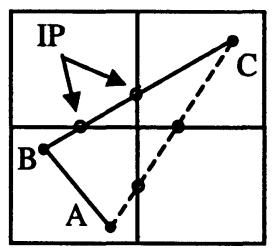

${ }^{4}$ Crosses means travelling from one pixel into another, touching on a pixel side or vertex does not count as crossing. 
Figure $7 \mathrm{~B}$ is not removed as $\mathrm{AC}$ will render different pixels to $\mathrm{AB}$ and $\mathrm{BC}$ together.

To ensure that the shape of the polyline is not compromised the PG method automatically retains all entering and exiting lines for each pixel. Another benefit to this is that is creates the base of a hierarchical data structure. The data can thus be used for future image manipulation that is commonly used in digital cartography; i.e. zooming in and out, and panning.

\subsection{Minimal Perfect Generalization}

Given $R$ from the PG algorithm we wish to further reduce it by eliminating points that are not crucial to preserve the visual quality of the image. The MPG simplification follows the definition given in Section 3 that the simplified line must only occupy or cross pixels at $r$ ' containing pixels that are originally rendered at $r$.

The point sequence is processed from head to tail. Given a point $p_{i}$ and another point $p_{n}$ where $n>i+1$ all intermediate points $p_{m}$, where $i<m<n$, are checked for possible elimination. To do so we must ensure that the set of pixels crossed by $p_{i}-p_{n}$ is equal to the cumulative set of pixels crossed by all line segments that occur between $p_{i}$ and $p_{n}$. Or $\mathrm{P}\left(p_{i}-p_{n}\right)=\mathrm{P}\left(p_{i}-p_{(i+1)}\right) \oplus \ldots \oplus$ $\mathrm{P}\left(p_{(n-1)}-p_{n}\right)$. To obtain the best simplification $p_{i}$ must be checked with each subsequent point to the end of the point sequence. The last pair $p_{i}-p_{n}$ that satisfies the above equality condition is taken as the simplified line segment. This reduction is maximised because there exists no point $p_{q}$ where $q>n$ such that $\mathrm{P}\left(p_{i}-p_{q}\right)=\mathrm{P}\left(p_{i}-p_{(i+1)}\right) \oplus \ldots \oplus \mathrm{P}\left(p_{(q-1)}-p_{q}\right)$. The process is then repeated beginning with $p_{n}$. The process ends when $p_{n}$ is the last point in the sequence. The result is a simplified polyline $S$. If the polyline is originally open then the MPG is complete. However if the object is a closed polygon then the first point $p_{i}$ has not been checked and may not be needed. Starting with the endpoint $p_{n}$ a final pass is performed on the now simplified point sequence $S$ using the same equality condition mentioned earlier. One problem that arises from this approach is that MPG simplification $S$ is resultant from using $P_{i}$ as the starting point. Given any other starting point in the original sequence it is possible that a different simplification $S^{\prime}$ is produced that has fewer points than $S$.

This naive approach demands that every possible permutation of $S$ must be computed, and the smallest one chosen. However the added computation makes such an approach inefficient and leaves the door open for better solutions to MPG. 


\subsection{Perfect Visualization}

Once the simplified object $O^{\prime}$ is obtained it can be rendered to the screen. However, rendering points that are specified at the end resolution $r$ ' will not produce the desired effect of PG (figure 11b). Points from different simplifications may be located in different parts of a pixel, but when rendering the only information that can be passed to the rendering device is which pixels to render. This is because a pixel is the smallest definable graphical feature on the device, or a SVO. This defeats the purpose of PG retaining original points since it is the exact location of points within those pixels, and their connection to other such points, that determine the perfect generalization.

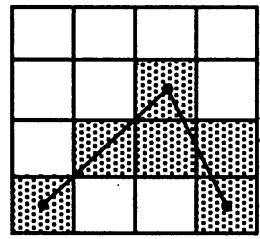

(a) A line simplified by the Li-Openshaw method.

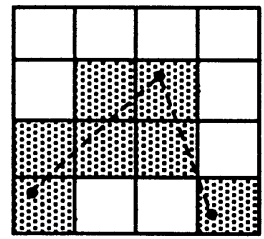

(b) The same line in (a) simplified by PG.

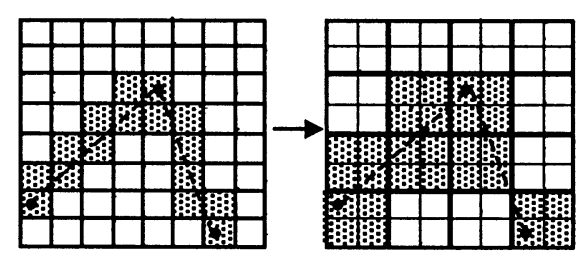

(c) Rendering of the

(d) Scaling of (c)

PG line in (b) at $r$. from $r$ (thin grid) to $r^{\prime}$ (thick grid).

Figure 8 The direct rendering to $r$ ' of a Li Openshaw simplification (a), and of an MPG simplification (b).

An extra processing step is needed in order for $O^{\prime}$ to be rendered accurately. The image must first be rendered at the original resolution, then be scaled to $r$ ' using some image scaling operation. Figure 11c illustrates a simple example of the MPG line displayed at a resolution double that of $r^{\prime 5}$. When scaled back to $r$ in 11d those pixels in which $r$ resolution pixels are contained are rendered. This results in the same pixels being rendered as those in figure $9 \mathrm{~b}$.

As a result of PG the shapes of individual objects are preserved as well as the final resolution r' will permit. That is objects occupy the same area they did originally except are represented by pixels of a coarser resolution covering this area. At $r^{\prime}$ these pixels are equivalent to a $\mathrm{Li}$ Openshaw SVO. It does not matter where in each SVO the original data was located, since anything inside a SVO is visually indistinguishable. The important thing is that all of the SVOs the object originally occupied are

\footnotetext{
${ }^{5}$ This resolution is used instead of the line's actual resolution only for display purposes, the latter being too fine to demonstrate with.
} 
retained. This is where the PG method differs to the Li Openshaw algorithm; the result will not omit areas originally covered (figure $3 b$ ) or cover new areas that did not contain the original data.

Topological relationships that exist in the same SVO, are technically not preserved since they are too small to display. Similarly relationships such as the disjointedness of two objects that occur in adjacent SVOs cannot be shown because the gap between them is too small (i.e. it at least needs 1 SVO between the object SVOs to be represented). In this sense topological relationships will only be kept if they are large enough to be visually significant on the display resolution. The full effect of PG on topological relationships required further study.

\section{CONCLUSIONS}

In this paper we identified that current line simplification algorithms were inadequate when the appearance of the generalization, rendered in a digital environments, must be considered.

We introduced the concepts of perfect generalization and minimal perfect generalization, designed with the specific goal of generalising spatial data to a compact solution without compromising visual quality. A way to achieve perfect generalization in linear time using standard spatial data is provided. These methods are targeted for use particularly in online (GIS) applications where hardware restrictions for efficient data reduction and accuracy. Simplification algorithms work on whole datasets, which are first fetched from some database. An alternate approach to improving efficiency is the possible use of spatial database structures (eg. indexes, z-values) and operations to reduce the data before it is sent for processing by generalization algorithms. The development of support mechanisms for this approach is a topic of further study.

When discussing generalization of an image at a particular scale to suit a certain resolution the density of data has a direct effect on the application of PG. In a worst case scenario very sparse data that cannot be reduced will be returned as it is by the algorithm, wasting both CPU and I/O resources. Conversely extremely dense data, where say an individual pixel has dozens of points, guarantees great reduction in storage requirements for an image. The question of effectiveness calls for testing of PG against other simplification techniques under differently distributed data. 


\section{ACKNOWLEDGMENTS}

This paper continues the work of Jackson Carroll's honours research on spatial object simplification in Spatial Data Simplification: The World is Just Too Much, (2000).

\section{REFERENCES}

[1] Barber C., Cromley R. \& Adrle R. (1995) Evaluating Alternative Line Simplification Strategies for Multiple Representations of Cartographic Lines, Cartographic and Geographic Information Systems, vol 22, no. 4, pp 276-290.

[2] Brassel K.E. \& Weibel R. (1988). A Review and Conceptual Framework of Automated Map Generalization, International Journal of Geographic Information Systems, 2(3), pp229-244.

[3] Douglas D.H. \& Peucker T.K. (1973) Algorithms for the Reduction of the Number of points Required to Represent a Digitized Line or its Caricature, The Canadian Cartographer, vol 10, no. 2, ppl12-122.

[4] Hoel E. \& Samet H. (1991) Efficient Processing of Spatial Queries in Line Segment Databases, Symposium on Large Spatial Databases, pp237-255.

[5] Horhammer M. \& Freeston M. (1999) Spatial Indexing With A Scale Dimension, Symposium on Large Spatial Databases, pp 52-71.

[6] Jansen, M. \& M. van Kreveld (1998) Evaluating the consistency of cartographic generalization, In T.K. Poiker and editors N. Chrisman, M., editors, Proceedings 8th International Symposium on Spatial Data Handling, pp. 668-678.

[7] Jones C.B (2001):

http://www.geog.utoronto.ca/gozdyra/teach/Lectures_GGR272/16_MapGeneralization.htm.

[8] Li Z. \& Openshaw S. (1992) Algorithms for Automated Line Generalization Based on a Natural Principle of Objective Generalization, International Journal of Geographical Information Systems, vol 6, no. 5, pp 373-389.

[9] McMaster R.B. (1983) A Mathematical Evaluation of Simplification Algorithms, Auto Carto 6 Proceedings, vol 2. pp 267-276.

[10] Peter \& Weibel (1999) Using Vector and Raster-Based Techniques in Cartographical Map Generalization, Third ICA Workshop on Progress in Automated Map Generalization, Ottawa.

[11] Ruas A. \& Plaznet C. (1996) Strategies for Automated Generalization, Proceedings of the $7^{\text {th }}$ International Symposium on Spatial Data Handling, vol 1, pp9A.1-9A.14.

[12] H. Samet. (1984) The Quadtree and Related Hierarchical Data Structures, ACM Computing Surveys, 16(2):187-260.

[13] Visvalingam \& Whyatt (1993) Line Generalization by Repeated Elimination of Points, The Cartographic Journal, vol 30, no.1, pp46-51.

[14] Weibel. R (1995) Map Generalization in the context of Digital Systems, Cartographic and Geographic Information Systems, Vol 22. pp259-263.

[15] Weibel R. (1996) A Typology of Constraints to Line Simplification, In Proceedings of the $7^{\text {th }}$ International Symposium on Spatial Data Handling, pp9A.1-9A.14. 


\section{BIOGRAPHY}

Sham Prasher is a PhD student in the department of Information Technology and Electrical Engineering at the University of Queensland. His research interests include spatial databases and geographic information systems. 